\title{
Artigos em Periódicos de Acesso Aberto: um Estudo com Pesquisadores Bolsistas de Produtividade do CNPq
}

\author{
Articles in Open Access Journals: a Study with Researchers awarded with \\ CNPq Productivity Fellowship
}

\section{Artículos en Periódicos de Acceso Abierto: un Estudio con Investigadores Becarios de Productividad del CNPq}

\author{
Jacqueline Leta | jleta@biogmed.ufrj.br \\ Universidade Federal do Rio de Janeiro, Programa de Educação, Gestão e Difusão em Ciências. Rio de Janeiro, Brasil. \\ Elaine Hipólito dos Santos Costa | elainebci04@gmail.com \\ Universidade Federal do Estado de São Paulo, Programa de Pós-Graduação em Ciência da Informação. Osasco, Brasil \\ Jesús Pascual Mena-Chalco | jesus.mena@ufabc.edu.br \\ Universidade Federal do ABC, Centro de Matemática, Computação e Cognição. Santo André, Brasil
}

\section{Resumo}

Historicamente, os periódicos eram editados e estavam sob a tutela, principalmente, de instituições acadêmicas e/ou científicas. Mais recentemente, editoras comerciais passaram a assumir esta atribuição, gerando custos adicionais à ciência, inclusive para se ter acesso às produções. A fim de romper com esta lógica surge, nos anos 2000, o Movimento de Acesso Aberto, que estimulou a criação de periódicos científicos de acesso aberto. Passadas quase duas décadas, o número de periódicos neste formato ultrapassa nove mil títulos, segundo o DOAJ. Considerando o papel deste tipo de periódico para a divulgação da pesquisa, o objetivo deste trabalho foi investigar se pesquisadores bolsistas de produtividade 1A do CNPq utilizam os periódicos de acesso aberto como estratégia para divulgar suas pesquisas. A análise de todos os artigos em periódicos, cadastrados na Plataforma Lattes, deste grupo seleto de pesquisadores revelou que parte significativa e crescente da produção do grupo está em periódicos de acesso aberto, sinalizando que uma fração destes notáveis atribui valor simbólico às publicações em periódicos de acesso aberto.

Palavras-chave: Acesso aberto; Periódicos científicos; Ciência Brasileira; Artigos científicos; Bourdieu. 


\section{Abstract}

Historically, scientific journals were edited and under responsibility, mainly, of academic or scientific institutions. More recently, private publishers assumed this task, what has generated additional costs to science, including to having access to publications. In order to disrupt this reasoning, in the 2000's, the Open Access Movement appeared and inspired the foundation of open access scientific journals. After almost two decades, the number of journals in open access format has exceeded nine thousand, according to DOAJ. Considering the role of such kind of journal in diffusing scientific research, the present study aimed to investigate whether researchers awarded with CNPq 1A productivity fellowship use open access journals as a strategy to diffuse their research. The analysis of all journal articles, listed in the Lattes Platform, authored by this particular team of researchers revealed that a relevant and growing part of their articles are in open access journals, an indication that a fraction of these notable researchers value such type of scientific journals.

Keywords: Open access; scientific journals; Brazilian science; scientific articles; Bourdieu.

\section{Resumen}

Históricamente los periódicos eran editados y estaban sobre la asistencia, principalmente, de instituciones académicas y/o científicas. Recientemente, editoras científicas comerciales asumieron esta atribución, generando gastos adicionales a la ciencia, inclusive para tener acceso a las publicaciones. Con la finalidad de quebrar con esta lógica, surge en los años 2000, el Movimiento de acceso Abierto, que estimuló la creación de revistas científicas de acceso abierto. Con mas de dos décadas, el número de revistas científicas en este formato sobrepasa nueve mil títulos, según el DOAJ. Considerando el papel de este tipo de revista científica para la divulgación de investigaciones, el objetivo de este trabajo es estudiar se investigadores con beca 1A por productividad en pesquisa del CNPq utilizan revistas de acceso abierto como estrategia para difundir sus investigaciones. El análisis de todos los artículos en revistas científicas, registrados en la Plataforma Lattes, de este grupo selecto de investigadores reveló que parte significativa y creciente de su producción está en revistas de acceso abierto, simbolizando que una fracción de estos notables asigna valor simbólico a las publicaciones en periódicos de acceso abierto.

Palabras clave: Acceso abierto; Revistas científicas; Ciencia Brasileña; Artículos científicos; Bourdieu.

\section{Introdução}

Ao longo dos últimos séculos, a comunicação científica tem sofrido diversas mudanças. Até o século XVII, os cientistas modernos utilizavam com freqüência a correspondência pessoal para a difusão de suas idéias e descobertas. Aos poucos, as sociedades científicas passaram a servir como o lugar onde os cientistas se encontravam para discutir experimentos e avaliar resultados, sendo os relatos e conclusões desses encontros, muitas vezes, registrados e distribuídos aos cientistas. Essa forma de divulgação influenciou o surgimento das primeiras revistas científicas em meados do século XVII, que, três séculos depois, se tornaram o principal meio de comunicação da difusão de novos conhecimentos na ciência. ${ }^{1}$

O processo definitivo para a incorporação das revistas como a principal forma de registro e comunicação da ciência só foi concluído no século XX, quando elas ganharam credibilidade, expressa principalmente através da revisão por pares, ou peer review, um processo em que o trabalho submetido passa pela avaliação e certificação de pares especialistas. Além deste aspecto, a redução do tempo de publicação e a maior facilidade na distribuição também contribuíram para a explosão do número de revistas científicas no mundo e, consequentemente, do número de trabalhos publicados.

Próximo à chegada do novo milênio, surgiram novas iniciativas para promover o livre acesso à informação científica, principalmente repositórios de auto-arquivamento de preprints como o ARXIV e o SSRN. Ações desta natureza se multiplicaram no mundo como uma resposta ao modelo de comunicação em periódicos que, nas últimas décadas, passou para o controle do setor privado, gerando custos adicionais 
à ciência, cientistas e suas instituições. Para Mueller², este cenário, de altos custos para publicar e acessar nos periódicos, também comprometeu o serviço de manutenção e atualização de coleções pelas bibliotecas norte-americanas e favoreceu o surgimento do Movimento de Acesso Aberto.

Já nos primeiros anos do século XXI, o Movimento de Acesso Aberto, cuja definição é "tornar disponível a qualquer internauta ler, fazer download, copiar, distribuir, imprimir, pesquisar ou referenciar o texto integral de artigos ou utilizá-los para outros fins legais sem qualquer barreira, desde que o trabalho seja devidamente reconhecido e citado"3, estimulou o surgimento de periódicos neste formato. Segundo Laakso et al 4, o movimento ocorreu em três fases: de 1993 a 1999 como os anos do "pioneirismo", de 2000 a 2004 os anos de estabilidade no crescimento e, depois desse período, uma queda no ritmo de crescimento. Fato é, no entanto, que o Movimento de Acesso Aberto permitiu o crescimento do número de periódicos, cujos conteúdos, ou seja, os artigos são abertos e gratuitos. Para o ano de 2017, o maior repositório de periódicos de Acesso Aberto, o Directory of Open Access Journals (DOAJ), mostra mais de nove mil títulos, de 129 países, em dezenas áreas do conhecimento.

Paralelamente ao crescimento dos periódicos de acesso aberto, a ciência e os cientistas do século XXI vivem o dilema de como lidar e de como se fazerem visíveis diante do grande volume de informação científico publicado anualmente. Em países de menor tradição no setor, aqueles ditos periféricos, como o Brasil, os cientistas são movidos e estimulados a buscar distinções e estratégias que os diferenciem. Para tal, reproduzem o modelo de ciência que é praticada em países de maior tradição, onde uma das ações mais estimuladas é a publicação em periódicos científicos renomados, indexados em bases de dados internacionais e que, em geral, não nasceram no formato aberto. Relevante ressaltar que a busca pela distinção na ciência passa pelo reconhecimento e legitimação dos pares, funcionando como uma espécie de capital simbólico ${ }^{5}$ e que, neste trabalho, é entendido como prestígio. Assim, a publicação em periódicos científicos, especialmente naqueles mais “cobiçados", é carregada de um simbolismo que se reflete, ao mesmo tempo, em maior reconhecimento pelos pares e em maior autoridade no campo, e, conseqüentemente, em maior poder dentro da estrutura hierárquica da ciência.

Considerando a expansão do modelo de periódico de acesso aberto e o momento atual da ciência, onde o cientista busca reconhecimento e prestígio através de suas publicações, o presente trabalho parte da questão: a elite de pesquisadores brasileiros utiliza os periódicos de acesso aberto como estratégia para divulgar suas pesquisas e, conseqüentemente, acumular prestígio? A fim de responder essa questão, utilizamos como insumo as informações bibliográficas, registradas na Plataforma Lattes, de todos os bolsistas de produtividade em pesquisa do CNPq, com vigência em 2016.

\section{Procedimento metodológico}

Com abordagem quantitativa, o trabalho tem como população de estudo os 1.205 pesquisadores bolsistas de produtividade 1A do CNPq, ativos no ano de 2016. A partir da lista com os nomes destes bolsistas, foi realizada a coleta de dados da produção científica, em formato de artigo em periódico, que estava listada no currículo destes pesquisadores na Plataforma Lattes, uma base de dados públicos que dá acesso a uma página na web onde o currículo do referido pesquisador fica hospedado.

A extração sistemática de diversos dados referentes a cada pesquisador, através do identificador único de acesso para cada CV (ID Lattes), foi realizada no dia 23 de maio de 2016 e, para esta etapa, utilizouse o Programa ScriptLattes ${ }^{6}$. A extração permitiu obter uma lista única (i. e., sem repetição) dos artigos completos publicados em periódicos científicos, com informações sobre o título da publicação, os nomes dos co-autores, o ano de publicação, o nome do periódico, o ISSN (ou e-ISSN) do periódico, número de páginas, volume e número do periódico. 
No mesmo dia, foram extraídas as informações de todos os periódicos de acesso aberto, disponíveis do site DOAJ. A consulta ao site permitiu obter uma lista completa dos títulos, assim como de todos os ISSNs e e-ISSNs dos periódicos declarados como acesso aberto. Posteriormente, foi realizado um cruzamento semi-automático dos dados referentes às publicações dos bolsistas e à lista de periódicos cadastrados no DOAJ, para identificar quais publicações estavam em periódicos de acesso aberto.

O cruzamento foi realizado em 2 etapas: Dado um ISSN (ou e-ISSN) de uma produção associada à lista de publicações dos bolsistas foi procurado o número na lista de ISSN do DOAJ. Caso o número não ser identificado foi realizada uma nova busca considerando o nome completo do periódico. Esta abordagem permite identificar publicações associadas a periódicos que mudaram seu ISSN ou que tiveram o número erroneamente cadastrado pelos bolsistas. Aqui é importante destacar que o casamento foi realizado após tanto os ISSNs quanto os nomes de revistas serem padronizados (i. e., os espaços em branco, hifens e pontos foram retirados dos ISSNs e os nomes dos periódicos foram transformados para minúsculas).

Todas as publicações em periódicos foram tabuladas, organizadas e discretizadas pelo ano de publicação, tipo (acesso restrito - que não consta da lista DOAJ - e acesso aberto) e grande área de conhecimento do bolsista (i.e., grande área que avalia e outorga a bolsa de produtividade ao pesquisador). Neste trabalho não consideramos informações sobre universidade, região ou gênero do pesquisador.

Finalmente, é importante frisar que, como os dados, aqui apresentados, referem-se à produção do conjunto de 1.205 pesquisadores bolsistas, todas as produções compartilhadas entre eles foram contabilizadas apenas uma vez, ou seja, os dados não têm dupla contagem.

\section{Resultados}

As informações extraídas da Plataforma Lattes referem-se aos artigos em periódicos dos 1.205 pesquisadores bolsistas de produtividade 1A currículos Lattes publicados em um período de 16 anos (janeiro de 2000 a dezembro de 2015) e são apresentadas em quatro quadriênios, o que permite verificar a dinâmica de produção deste grupo seleto de pesquisadores nos periódicos de acesso aberto. A Tabela 1 apresenta o total de artigos em periódicos e artigos em periódicos de acesso aberto dos bolsistas de produtividade $1 \mathrm{~A}$ ao longo de quatro quadriênios.

Tabela 1: Total de artigos em periódicos (A) e em periódicos de acesso aberto $(B)$ de pesquisadores bolsistas de produtividade 1 A, por quadriênio (2000-2015).

\begin{tabular}{l|l|l|l}
\hline Quadriênio & $\begin{array}{l}\text { Total de Artigos } \\
(\mathbf{A})\end{array}$ & $\begin{array}{l}\text { Artigos em Acesso } \\
\text { Aberto (B) }\end{array}$ & $\begin{array}{l}\text { \% Artigos em Acesso } \\
\text { Aberto (B/A) }\end{array}$ \\
\hline 2000 a 2003 & 23.832 & 4.827 & 20,3 \\
\hline 2004 a 2007 & 30.353 & 6.970 & 23,0 \\
\hline 2008 a 2011 & 34.851 & 8.867 & 25,4 \\
\hline 2012 a 2015 & 35.947 & 9.541 & 26,6 \\
\hline $\mathbf{2 0 0 0}$ a $\mathbf{2 0 1 5}$ & $\mathbf{1 2 4 . 9 8 3}$ & $\mathbf{3 0 . 2 0 5}$ & $\mathbf{2 4 . 2}$ \\
\hline
\end{tabular}

Fonte: Elaboração própria a partir das informações disponibilizadas nos Currículos Lattes. Não há dupla contagem.

Observa-se que, em termos absolutos, no período de 16 anos, houve um aumento significativo do total de produção em periódicos (de 23.832 para 35.947) e um aumento ainda maior na produção em periódicos de acesso aberto (de 4.827 para 9.541), o que se refletiu na fração que esta forma de publicação representa para o total da produção deste grupo (de 20,3\% para 26,6\%). Os dados indicam, portanto, que o grupo como um todo está publicando cada vez mais no formato acesso aberto. 
A Tabela 2 mostra a distribuição, por quadriênio, da fração de artigos em acesso aberto dos bolsistas 1A em cada grande área do conhecimento. Importante destacar que cada percentual representa a divisão do total de artigos em acesso aberto de uma grande área, num dado quadriênio, pelo total de artigos da mesma grande área no mesmo quadriênio. Desta forma, os percentuais são relativos às grandes áreas do conhecimento.

Tabela 2. Artigos em acesso aberto (\%) em cada grande área dos pesquisadores bolsistas de produtividade 1 A, por quadriênio (2000-2015)

\begin{tabular}{l|l|l|l|l}
\hline $\begin{array}{l}\text { Grandes áreas do } \\
\text { conhecimento }\end{array}$ & $\mathbf{2 0 0 0}$ a $\mathbf{2 0 0 3}$ & $\mathbf{2 0 0 4}$ a $\mathbf{2 0 0 7}$ & $\mathbf{2 0 0 8}$ a $\mathbf{2 0 1 1}$ & $\mathbf{2 0 1 2}$ a $\mathbf{2 0 1 5}$ \\
\hline Ciências Agrárias & $44,71 \%$ & $48,23 \%$ & $47,10 \%$ & $36,45 \%$ \\
\hline Ciências Biológicas & $12,34 \%$ & $12,73 \%$ & $16,57 \%$ & $25,97 \%$ \\
\hline Ciências Exatas e da Terra & $5,81 \%$ & $7,41 \%$ & $10,10 \%$ & $10,45 \%$ \\
\hline Ciências Humanas & $30,10 \%$ & $37,13 \%$ & $38,75 \%$ & $37,56 \%$ \\
\hline Ciências Sociais Aplicadas & $17,38 \%$ & $28,96 \%$ & $33,94 \%$ & $42,10 \%$ \\
\hline Ciências da Saúde & $26,72 \%$ & $28,96 \%$ & $30,68 \%$ & $32,98 \%$ \\
\hline Engenharias & $10,87 \%$ & $11,60 \%$ & $12,76 \%$ & $14,44 \%$ \\
\hline Lingüística, Letras e Artes & $11,58 \%$ & $14,82 \%$ & $17,19 \%$ & $17,43 \%$ \\
\hline Outra & $8,99 \%$ & $12,01 \%$ & $16,73 \%$ & $29,01 \%$ \\
\hline
\end{tabular}

Fonte: Elaboração própria a partir das informações disponibilizadas nos Currículos Lattes. Os percentuais representam o seguinte cálculo: ( $n$ ) publicações de acesso aberto na grande área $X$, em um quadriênio, dividido por ( $n$ ) publicações totais da grande área $X$, no mesmo quadriênio.

Observa-se que, ao longo dos quadriênios, a fração de artigos em periódicos de acesso aberto dos pesquisadores bolsistas 1A, de sete das nove grandes áreas, aumentou; as exceções são as publicações dos pesquisadores das ciências agrárias e das humanas. Apesar desta redução, os pesquisadores destas duas áreas estão entre os com maior fração de publicações em acesso aberto. Artigos de pesquisadores das ciências biológicas e das exatas tiveram um aumento, no período, bastante significativo, de quase duas vezes. O maior aumento foi nas produções de pesquisadores de outras grandes áreas, que incluem, dentre outras, bolsistas das áreas de Biomedicina, Química industrial, Relações internacionais, Ciências atuariais, e Engenharia mecatrônica.

\section{Discussão e Considerações finais}

A partir desta análise, foi possível observar que uma fração significativa e crescente das produções em formato de artigo deste grupo seleto da ciência brasileira, cerca de $25 \%$ no período, está em periódicos de acesso aberto. Os dados apontam que o grupo, como um todo, vem usando a produção neste formato, o acesso aberto, para divulgar suas pesquisas e, consequentemente, como um mecanismo para acumular prestígio ou capital.

O cenário positivo e promissor sobre a produção em acesso aberto da elite da ciência brasileira se contrasta, aparentemente, com dados recentes apresentados em relatório da Comissão Européia sobre a produção em acesso aberto em diversos países ${ }^{7}$. Segundo o relatório, 66\% da produção brasileira (do total de 27.158 artigos) está em acesso aberto, uma fração maior que o observado para conjunto de 28 países que compõem a União Européia (51\%) e para outros países, como Canadá (56\%), EUA (59\%) e Japão (44\%).

Importante destacar que o relatório a Comissão Européia considerou para análise: o período de 2008 a 2013 e as produções foram classificadas de acesso aberto desde que estivessem disponíveis on-line, sem 
custo e/ou qualquer outra barreira para o usuário. Já o presente estudo focou na produção da elite da ciência brasileira em um período maior (2000 a 2015) e utilizou a lista de periódicos do DOAJ para classificar as produções do grupo de pesquisadores bolsistas. Diferente do trabalho internacional, no presente estudo não foram consideradas as produções de periódicos híbridos (aqueles de acesso restrito que disponibilizam os artigos on-line mediante pagamento de taxa).

A despeito destas diferenças, a alta fração de artigos em acesso aberto, encontrada para o Brasil, no estudo a Comissão Européia, não deve ser ignorada. Trata-se de um resultado sobre uma produção que tem autoria muito diversificada, incluindo pesquisadores bolsistas de diferentes níveis e, principalmente, pesquisadores não bolsistas. Considerando, então, os dois resultados, é possível pensar que exista uma relação inversamente proporcional entre o nível do bolsista e as frações de produção em periódicos de acesso aberto: quanto maior o nível, menor a fração. Esse modelo pode ser inserido nas reflexões de Bourdieu sobre capital e campo; pesquisadores de maior prestígio, como os bolsistas 1A, tendem a publicar em periódicos científicos certificados, que, de modo geral, não estão na lista DOAJ. Isso garante a eles maior reconhecimento e legitimação dos pares, que se expressam em maior autoridade e poder dentro do campo que atuam. Por outro lado, pesquisadores bolsistas em níveis abaixo do $1 \mathrm{~A}$ e, principalmente, não bolsistas estariam mais abertos a publicar em periódicos de menor prestígio, os menos "cobiçados", incluídos, mais freqüentemente, na lista DOAJ, como uma estratégia de se estabelecer no campo.

É necessário, também, atentar para o efeito de outros fatores no processo de escolha do formato do periódico (aberto ou não). É possível que pesquisadores de determinadas áreas, por exemplo, preferiram periódicos de acesso aberto internacionais, outros buscam aqueles com cobrança para publicação. É possível que a região geográfica da instituição de vínculo dos pesquisadores também influencie nesta escolha. Para melhor compreender a dinâmica do publicar em acesso aberto neste grupo seleto de pesquisadores, análises complementares que partem de algumas variáveis, como as citadas anteriormente, estão sendo realizadas.

Por fim, vale notar que os dados apresentados neste trabalho são, até onde sabemos, pioneiros para a ciência brasileira e fazem parte de um projeto maior, que busca entender a produção em acesso aberto nos diferentes extratos dos pesquisadores brasileiros. Trata-se, portanto, de um foco distinto da literatura existente, até o momento, no tema (cuja ênfase é na produção total dos países, inclusive do Brasil ${ }^{6}$ ) e que poderá revelar tendências muito específicas de como a ciência brasileira se estrutura em torno deste novo e crescente formato de produção, o acesso aberto.

\section{Referências}

1. Meadows AJ, Meadow CT. Communicating research. San Diego, CA: Academic Press; 1998.

2. Mueller SPM. Produção e financiamento de Periódicos Científicos de Acesso Aberto: um estudo na base SciELO. In: Población DA, et al (Org.) Revistas científicas: dos processos tradicionais às perspectivas alternativas de comunicação. São Paulo: Ateliê Editorial ; 2011 : 201-230.

3. Budapest Open Access Initiative [Internet] ; 2002.

4. Laakso M, et al. The development of open access journal publishing from 1993 to 2009. PloS one [Internet]. 2011 [citado em 14 dez. 2015]; 6(6) : e20961.Disponível em: http://journals.plos.org/ plosone/article?id=10.1371/journal.pone.0020961

5. Bourdieu P. Os usos sociais das ciências: por uma sociologia clínica do campo científico. São Paulo: UNESP; 2003.

6. Mena-Chalco JP, Cesar Junior RM. ScriptLattes: an open source knowledge extraction system from the Lattes platform. Journal of the Brazilian Computer Society, 2009; 15(4) : 31-39.

7. Archambault $\mathrm{E}$, et al. Proportion of open access papers published in peer-reviewed journals at the European and world levels1996-2013. 2014.[Internet]. Disponível em: http://science-metrix.com/sites/ default/files/science-metrix/publications/d $1.8 \mathrm{sm} \mathrm{ec} \mathrm{dg-rtd} \mathrm{proportion} \mathrm{oa} \mathrm{1996-2013} \mathrm{v11p.pdf}$ 